\title{
Parameterless discrete Painlevé equations and their Miura relations
}

B. Grammaticos, A. Ramani

To cite this article: B. Grammaticos, A. Ramani (2016) Parameterless discrete Painlevé equations and their Miura relations, Journal of Nonlinear Mathematical Physics 23:1, 141-149, DOI: https://doi.org/10.1080/14029251.2016.1135647

To link to this article: https://doi.org/10.1080/14029251.2016.1135647

Published online: 04 January 2021 


\title{
Parameterless discrete Painlevé equations and their Miura relations
}

\author{
B. Grammaticos \\ IMNC, Université Paris VII \& XI, CNRS, UMR 8165 \\ Bât. 440, 91406 Orsay, France \\ grammaticos@imnc.in2p3.fr \\ A. Ramani \\ Centre de Physique Théorique, Ecole Polytechnique \\ CNRS, Université Paris-Saclay, 91128 Palaiseau, France \\ Alfred.Ramani@cpht.polytechnique.fr
}

Received 21 October 2015

Accepted 1 December 2015

\begin{abstract}
We present a study of discrete Painlevé equations which do not have any parameter, apart from those that can be removed by the appropriate scaling. We find four basic equations of this type as well as several more related to the basic ones by Miura transformations, which we derive explicitly. We obtain also the continuous limits of the basic parameterless equations and show that two of them are the discrete analogues of both the continuous Painlevé I and the zero-parameter Painlevé III.
\end{abstract}

Keywords: discrete Painlevé equations, Miura transformations, continuous limits.

PACS numbers: 02.30.Ik, 05.45.Yv

\section{Introduction}

Before proceeding further we must make clear what is meant by term "parameterless" used in the title of the paper. A parameterless discrete, or continuous, Painlevé equation is an equation where, after all possible scalings have been applied in order to remove spurious parameters, there remains no genuine free parameter in the equation and none can be introduced by auto-Bäcklund/Schlesinger transformations. Let us give a few examples based on the continuous case [1]. The obvious choice of a parameterless Painlevé equation is $\mathrm{P}_{\mathrm{I}}$

$$
u^{\prime \prime}=6 u^{2}+t
$$

On the other hand $\mathrm{P}_{\mathrm{II}}$ with the parameter put to 0 , i.e.

$$
u^{\prime \prime}=2 u^{3}+t u
$$

is not a parameterless equation since the application of an auto-Bäcklund transformation can bring back the missing parameter. The case of $\mathrm{P}_{\mathrm{III}}$

$$
u^{\prime \prime}=\frac{u^{\prime 2}}{u}-\frac{u^{\prime}}{t}+\frac{1}{t}\left(\alpha u^{2}+\beta\right)+\gamma u^{3}+\frac{\delta}{u}
$$

is more interesting [2]. When all four visible parameters are non-zero one can use a scaling of the dependent and independent variable in order to put, say, $\gamma=1$ and $\delta=-1$, leading to two genuine 
parameters $\alpha$ and $\beta$. This is the case of the full, two-parameter, $\mathrm{P}_{\mathrm{III}}$. When $\gamma=0$ and $\alpha \delta \neq 0$ we have the case of the one-parameter $\mathrm{P}_{\mathrm{III}}$ studied in [3]. When $\gamma=\delta=0$ with $\alpha \beta \neq 0$ we have a case with no genuine parameter, since a scaling can be used in order to put $\alpha=-\beta=1$. This is a zero-parameter subcase of $\mathrm{P}_{\mathrm{III}}$. However the solutions of this equation do not introduce a new transcendent. Indeed, the change of variables $t=s^{2}$ and $u=v^{2}$ transforms the equation to one where $\alpha=\beta=0$ with $\gamma \delta \neq 0$ whereupon auto-Bäcklund transformations can be used in order to regenerate the missing parameters. Thus the solutions of the zero-parameter, $\gamma=\delta=0$, case can be expressed in terms of the solutions of the full $\mathrm{P}_{\mathrm{III}}$. This last statement needs some clarification. In [4] Ohyama and collaborators studied the Painlevé III equation and concluded that the $\gamma=\delta=0$ case is different from the $\gamma \delta \neq 0$ one. This is indeed true when one considers only transformations of homographic type which allow to derive the various equivalent forms of Painlevé equations, as explained in the seminal paper of Gambier [5]. However in the present case we perform a transformation of different type, a folding transformation in the terminology of [6], which indeed allow us to express the solutions of the zero-parameter $\mathrm{P}_{\text {III }}$ in terms of the solutions of the full one. The case $\alpha=\gamma=0$, with $\beta \delta \neq 0$ is another parameterless case. However in that case a transformation of both dependent and independent variables, $t=e^{s}$ and $u=t v$, can transform the equation to a purely autonomous one for $v$, solvable in terms of elliptic functions. Finally when only one (or none) of the four parameters is non-zero we have an equation the solution of which is elementary.

The situation is more complicated in the case of discrete Painleve equations. The first remark is that the discrete Painleve equations come in three "flavours", additive, multiplicative and elliptic where the dependence on the independent variable enters polynomially, exponentially or through the argument of an elliptic function respectively. While the third type of discrete Painlevé equations corresponds to systems with 8 parameters, we may encounter parameterless instances of discrete Painlevé equations of both additive and multiplicative type. Sakai, in his celebrated work of classification of discrete Painlevé equations in terms of surfaces [7], has presented the complete degeneration pattern whereupon one starts from 8-parameter equations associated to the affine Weyl group $E_{8}^{(1)}$ and, by a process of coalescence, ends up with parameterless equations associated to the various groups $A_{1}^{(1)}$. It turns out that there exist four such groups, two corresponding to additive equations and two corresponding to multiplicative ones. Thus one would normally expect four parameterless discrete Painlevé equations to exist.

The situation is even more complicated, and in fact more such equations have been found over the years. The explanation of this is that there exist transformations that allow to transform a given discrete Painlevé equation into another one with a perfectly canonical aspect. The aim of this paper is to propose as complete as possible a list of these discrete Painlevé equations and derive the Miura transformations which link them together. While some of these parameterless equations have been known for many years there exist others which will be presented here for the first time.

\section{Derivation of the basic parameterless discrete Painlevé equations}

In his classification of discrete Painlevé equations, Sakai presented the degeneration pattern from which one can deduce that four different parameterless discrete Painlevé equations can be obtained by applying the adequate limiting procedure on equations associated to the affine Weyl group $A_{1}^{(1)}+$ $A_{1}^{(1)}$. We shall refer to these equations as the basic parameterless ones. Since there exist several (in fact, infinitely many) equations within $A_{1}^{(1)}+A_{1}^{(1)}$ it is important to choose the right one in order to derive the corresponding equation in $A_{1}^{(1)}$. We start by deriving the additive equations. The 
equations related to the group $A_{1}^{(1)}+A_{1}^{(1)}$ are multiplicative and thus a limit of the independent variable is mandatory. In what follows we shall use the following notational convention. We shall write the equations in $A_{1}^{(1)}+A_{1}^{(1)}$ in upper case symbols and reserve the lower case ones for the equations in $A_{1}^{(1)}$. Thus $Z_{n}$ must be understood as $Z_{n}=Z_{0} Q^{n}$ while for the additive equations we have $z_{n}=\alpha n+z_{0}$.

We start from

$$
\left(X_{n} X_{n+1}-1\right)\left(X_{n} X_{n-1}-1\right)=\frac{A Z_{n}^{2} X_{n}}{X_{n}-Z_{n}}
$$

and introduce the ansatz $X_{n}=1+\varepsilon x_{n}, Z_{n}=1+\varepsilon z_{n}$ (which means that $Q=1+\varepsilon \alpha$ ) and take $A=\varepsilon^{3}$. By taking the limit $\varepsilon \rightarrow 0$ we find the equation

$$
\left(x_{n}+x_{n+1}\right)\left(x_{n}+x_{n-1}\right)=\frac{1}{x_{n}-z_{n}}
$$

which was first obtained in [8]. Next we start from the equation, introduced in [3] as the oneparameter $q$ - $\mathrm{P}_{\mathrm{III}}$ :

$$
\begin{gathered}
X_{n} X_{n+1}=\frac{Y_{n}\left(Y_{n}+1\right)}{A\left(1+Z_{n} Y_{n}\right)} \\
Y_{n} Y_{n-1}=A X_{n}\left(1+X_{n}\right)
\end{gathered}
$$

and use the ansatz $X_{n}=-1 / 2+\varepsilon x_{n} / 2, Y_{n}=-1-\varepsilon^{2} y_{n}, Z_{n}=1+\varepsilon^{3} z_{n}, A=-4-4 \varepsilon^{2}$ and find at the limit $\varepsilon \rightarrow 0$ the equation

$$
\begin{gathered}
x_{n}+x_{n+1}=\frac{z_{n}}{y_{n}} \\
y_{n}+y_{n-1}=-x_{n}^{2}+1
\end{gathered}
$$

which can be combined into a single equation for $x$

$$
\frac{z_{n}}{x_{n}+x_{n+1}}+\frac{z_{n-1}}{x_{n}+x_{n-1}}=-x_{n}^{2}+1
$$

This is the well-known alternate discrete form of $\mathrm{P}_{\mathrm{I}}$, initially obtained in [9] as the contiguity relation of the solutions of the continuous Painlevé II equation.

We turn now to the multiplicative parameterless equations. Here the meaning of $z_{n}$ has changed with respect to the previous paragraph. We have now $z_{n}=z_{0} q^{n}$. We start from the equation obtained in [3] and which is related to the one-parameter $q-\mathrm{P}_{\mathrm{III}}$ :

$$
\begin{aligned}
& \left(X_{n+1} Y_{n}-1\right)\left(X_{n} Y_{n}-1\right)=Z_{n} Y_{n}\left(1+Y_{n}\right) \\
& \left(X_{n} Y_{n}-1\right)\left(X_{n} Y_{n-1}-1\right)=A Z_{n}\left(1+X_{n}\right)
\end{aligned}
$$

and consider the limit $X_{n}=\varepsilon x_{n}, Y_{n}=y_{n} / \varepsilon, Z_{n}=z_{n} \varepsilon^{2}, A=1 / \varepsilon^{2}$ for $\varepsilon \rightarrow 0$ leading to

$$
\left(x_{n+1} y_{n}-1\right)\left(x_{n} y_{n}-1\right)=z_{n} y_{n}^{2}
$$




$$
\left(x_{n} y_{n}-1\right)\left(x_{n} y_{n-1}-1\right)=z_{n}
$$

This equation was first presented in [10]. Finally we use another equation derived in [11] are which is equivalent to (4):

$$
\begin{aligned}
& X_{n} X_{n+1}=\frac{Z_{n}}{1+Y_{n}} \\
& Y_{n} Y_{n-1}=\frac{A Z_{n}}{1+X_{n}}
\end{aligned}
$$

and consider the limit $Y_{n}=y_{n} / \varepsilon, X_{n}=x_{n}, Z_{n}=z_{n} / \varepsilon, A=1 / \varepsilon$ when $\varepsilon \rightarrow 0$. The first equation gives readily $y_{n}=z_{n} /\left(x_{n} x_{n+1}\right)$ whereupon substituting in the second equation we find,

$$
x_{n+1} x_{n-1}=z_{n-1}\left(\frac{1}{x_{n}}+\frac{1}{x_{n}^{2}}\right)
$$

This equation is the $q$-discrete $\mathrm{P}_{\mathrm{I}}$ albeit in non-canonical form. However it suffices to introduce the gauge $x_{n} \rightarrow \zeta_{n} x_{n}$ with $\zeta_{n}=z_{n-1}^{1 / 4}$ in order to bring (12) to the canonical form

$$
x_{n+1} x_{n-1}=\frac{\zeta_{n}}{x_{n}}+\frac{1}{x_{n}^{2}}
$$

Equations (5), (8), (10) and (13) are the basic parameterless discrete Painlevé equations related to the four different $A_{1}^{(1)}$ affine Weyl groups in the Sakai classification.

\section{Miura transformations}

a) Additive equations

No Miura transformation is known for the alternate discrete Painlevé I equation, (8). On the other hand equation (5) is connected to another well-known discrete form of Painlevé I as we have shown in [12]. Indeed, the Miura transformation

$$
\begin{gathered}
w_{n+1} w_{n}=x_{n}-z_{n} \\
x_{n}+x_{n-1}=\frac{1}{w_{n}}
\end{gathered}
$$

leads to (5) upon elimination of $w$, while, when we eliminate $x$ we obtain

$$
w_{n+1}+w_{n-1}=\frac{1}{w_{n}^{2}}-\frac{z_{n}+z_{n-1}}{w_{n}}
$$

an equation which is in fact the contiguity relation of the solutions of the one-parameter continuous $\mathrm{P}_{\mathrm{III}}[12]$. 
b) Multiplicative equations

We start by introducing the Miura transformation

$$
\begin{gathered}
x_{n+1} x_{n}=z_{n} w_{n} \\
w_{n} w_{n-1}=1+x_{n}
\end{gathered}
$$

Eliminating $w$ between the two leads to

$$
x_{n+1} x_{n-1}=z_{n} z_{n-1}\left(\frac{1}{x_{n}}+\frac{1}{x_{n}^{2}}\right)
$$

i.e. essentially the $q-\mathrm{P}_{\mathrm{I}}$ equation (12) obtained in the previous paragraph. On the other hand eliminating $x$ we find

$$
\left(w_{n} w_{n+1}-1\right)\left(w_{n} w_{n-1}-1\right)=z_{n} w_{n}
$$

and equation first identified in [8].

Next we turn to the Miura of equation (10). We introduce

$$
\begin{gathered}
x_{n} y_{n}=w_{n}+1 \\
x_{n} y_{n-1}=u_{n}+1 \\
w_{n} u_{n+1}=z_{n} y_{n}^{2} \\
w_{n} u_{n}=z_{n}
\end{gathered}
$$

which is nothing but a reparametrisation of (10), meaning that $u$ and $w$ can be eliminated in a straightforward way leading to (10) for $x$ and $y$. Now using (19d) we can express $u$ in terms of $w$ and from (19a) and (19b) we can obtain for $y$ the equation $y_{n} / y_{n-1}=w_{n}\left(1+w_{n}\right) /\left(w_{n}+z_{n}\right)$. It suffices now to take the square of the latter and use (19c) in order to eliminate $y$. Finally expressing everything in terms of $w$ we find

$$
w_{n+1} w_{n-1}=\frac{z_{n+1} z_{n-1}}{z_{n}^{2}} \frac{\left(w_{n}+z_{n}\right)^{2}}{\left(w_{n}+1\right)^{2}}
$$

and, given that $z_{n}=z_{n} q^{n}$, the prefactor in the right-hand side of (20) is equal to 1. Equation (20) was first obtained in [13] where we have derived quadratic relations, i.e. folding [6] transformations, between the solutions of Painlevé equations, both continuous and discrete. However its relation to (10) was not noticed at the time (for the simple reason that the latter had yet to be derived).

We turn now to two parameterless equations the derivation of which has been made possible by our recent extension of the study of discrete Painlevé equations to what we called strongly asymmetric forms [14]. The latter refer to a system of two equations the right-hand sides of which 
have different functional forms and which, as a consequence, cannot be easily obtained by the deautonomisation of a symmetric QRT mapping [15]. The first of the equations in question is

$$
\begin{gathered}
\left(x_{n+1} y_{n}-1\right)\left(x_{n} y_{n}-1\right)=\frac{1}{1+y_{n} / z_{2 n}} \\
\left(x_{n} y_{n}-1\right)\left(x_{n} y_{n-1}-1\right)=z_{2 n-1} x_{n}^{3}
\end{gathered}
$$

where we have introduced the index $2 n$ in the independent variable anticipating the transformations introduced below. In the present case we do not have a standard Miura transformation i.e. a system where one can eliminate either of the variables leading to the respective equations, but a more complicated situation. We start from

$$
\begin{gathered}
x_{n} w_{2 n} w_{2 n-1}=1 \\
y_{n}=-z_{2 n}+w_{2 n+2} w_{2 n+1}^{2} w_{2 n}^{2} w_{2 n-1}
\end{gathered}
$$

We remark readily that, while the products $w_{2 n+2} w_{2 n+1}$ and $w_{2 n} w_{2 n-1}$ entering the expression of $y$ can be respectively expressed in terms of $x_{n+1}$ and $x_{n}$, this is not the case for the product $w_{2 n+1} w_{2 n}$ which can only be obtained through $y$. Still we make the assumption that there exists an equation for $w_{n}$ valid for every value of $n$ and compatible with the equation (21) and the transformations (22). We find that this is indeed the case provided $w$ obeys the equation

$$
w_{n+1} w_{n-1}=\frac{\zeta_{n}}{w_{n}}+\frac{1}{w_{n}^{2}}
$$

i.e. the $q-\mathrm{P}_{\mathrm{I}}$ equation already encountered and where $\zeta$ and $z$ are related through $z_{2 n}=\zeta_{n} \zeta_{n+1}$.

The second parameterless equation is

$$
\begin{aligned}
& \left(x_{n+1} y_{n}-1\right)\left(x_{n} y_{n}-1\right)=\frac{z_{3 n}^{2} y_{n}^{2}}{y_{n}+z_{3 n}} \\
& \left(x_{n} y_{n}-1\right)\left(x_{n} y_{n-1}-1\right)=\frac{z_{3 n} z_{3 n-3}}{x_{n}}
\end{aligned}
$$

where, again, the indices of $z$ were adequately introduced. Here the transformation is

$$
\begin{gathered}
x_{n}=w_{3 n-1} w_{3 n-2} \\
y_{n}=w_{3 n}
\end{gathered}
$$

Again, we assume that an equation for $w$, valid for every $n$, does exist which leads to

$$
w_{n+1} w_{n-1}=\frac{1}{w_{n}}+\frac{z_{n}}{w_{n}^{2}}
$$

This is again the $q$ - $\mathrm{P}_{I}$ equation, in non-canonical form. A gauge transformation can be introduced at this stage $w_{n} \rightarrow \zeta_{n}^{-1 / 3} w_{n}$ with $\zeta_{n}=z_{n}^{-3 / 4}$ bringing (26) to the canonical form

$$
w_{n+1} w_{n-1}=\frac{\zeta_{n}}{w_{n}}+\frac{1}{w_{n}^{2}}
$$

Thus the two new parameterless equations are related to the same $q$-Painlevé I. 


\section{Continuous limits}

In this section we shall present the continuous limits of the basic parameterless discrete Painlevé equations. We shall work with the systems obtained in section 3 with one exception: instead of equation (5) we shall work with (15) since the latter is a more customary form.

We start from

$$
\frac{z_{n}}{x_{n}+x_{n+1}}+\frac{z_{n-1}}{x_{n}+x_{n-1}}=-x_{n}^{2}+1
$$

and introducing the ansatz $x=\left(1+\varepsilon^{2} u\right) / \sqrt{3}, z=\left(4+\varepsilon^{4} t\right) /(6 \sqrt{3})$ we obtain, at the limit $\varepsilon \rightarrow 0$, the Painlevé I equation $u^{\prime \prime}=6 u^{2}+t$, as expected.

Next we consider the limit of

$$
\begin{gathered}
\left(x_{n+1} y_{n}-1\right)\left(x_{n} y_{n}-1\right)=z_{n} y_{n}^{2} \\
\left(x_{n} y_{n}-1\right)\left(x_{n} y_{n-1}-1\right)=z_{n}
\end{gathered}
$$

Introducing the ansatz $y=1-\varepsilon v, x=1+\varepsilon v+\varepsilon^{2}\left(v^{2}+e^{t} u\right)$ and $z=\varepsilon^{4} e^{2 t}$ we find at the limit $\varepsilon \rightarrow 0$ $v=u^{\prime} /(2 u)-1 / 2$ and finally the equation

$$
u^{\prime \prime}=\frac{u^{\prime 2}}{u}-2 e^{t}\left(u^{2}-1\right)
$$

which is precisely the zero-parameter Painlevé III equation.

Things are becoming more interesting in the case of the two remaining equations. We start from the equation

$$
x_{n+1}+x_{n-1}=\frac{z_{n}}{x_{n}}+\frac{a}{x_{n}^{2}}
$$

where we have introduced, through scaling, the parameter $a$ so as to avoid denominators in the ansatz. The latter takes the form $x=-1+\varepsilon^{2} u, z=6-\varepsilon^{4} t$ with $a=4$. Taking the limit $\varepsilon \rightarrow 0$ we obtain indeed the Painlevé I equation $u^{\prime \prime}=6 u^{2}+t$. However another limit is possible. We start by considering two instances of (31) by separating the variables of even and odd indices. We have thus $v_{n}=x_{2 n}$ and $y_{n}=x_{2 n+1}$ and the equation is rewritten as

$$
\begin{gathered}
v_{n+1}+v_{n}=\frac{z_{2 n+1}}{y_{n}}+\frac{a}{y_{n}^{2}} \\
y_{n}+y_{n-1}=\frac{z_{2 n}}{v_{n}}+\frac{a}{v_{n}^{2}}
\end{gathered}
$$

We introduce the ansatz $y=t / u+\varepsilon\left(u-t u^{\prime}\right) /\left(2 u^{2}\right)+\varepsilon^{2} /\left(4 u^{2}\right), a=\varepsilon^{2} / 2$ while, at the limit $\varepsilon \rightarrow 0$, $v$ and $z$ go over to the continuous variables $u$ and $t$. We obtain thus the equation

$$
u^{\prime \prime}=\frac{u^{\prime 2}}{u}-\frac{u^{\prime}}{t}+\frac{1}{t^{2}}\left(u^{2}-t\right)
$$

which is the zero parameter $\mathrm{P}_{\mathrm{III}}$ equation (albeit in non-canonical form). 
A similar situation arises in the case of the multiplicative equation

$$
x_{n+1} x_{n-1}=\frac{z_{n}}{x_{n}}+\frac{a}{x_{n}^{2}}
$$

Introducing the ansatz $x=1-\varepsilon^{2} u, z=4-\varepsilon^{4} t$ with $a=-3$ we find, at the limit $\varepsilon \rightarrow 0$ the Painlevé I equation, (1), just as in the case of the additive equation above. Here again another limit is possible. We consider again two instances of the equation based on the parity of the indices: $v_{n}=x_{2 n}$ and $y_{n}=1 / x_{2 n+1}$. The system, now with $a=1$, becomes

$$
\begin{gathered}
v_{n+1} v_{n}=z_{2 n+1} y_{n}+y_{n}^{2} \\
y_{n} y_{n-1}=\frac{v_{n}^{2}}{1+z_{2 n} v_{n}}
\end{gathered}
$$

Next we introduce the ansatz $y=u+\varepsilon u^{\prime} / 2+\varepsilon^{2}\left(u^{\prime 2} /(8 u)-e^{t} u^{2} / 2\right)$ and $z=\varepsilon^{2} e^{t}$ while, at the limit $\varepsilon \rightarrow 0, v$ goes over to the continuous variable $u$. The resulting equation is a zero-parameter $\mathrm{P}_{\mathrm{III}}$ and, in fact, precisely (30).

Thus while among the four basic parameterless equations there exist two which can be labeled as discrete Painlevé I and $q$-discrete zero-parameter Painlevé III respectively, there exist two equations which play the role of discrete analogues of both parameterless continuous Painlevé equations.

\section{Conclusions}

In this paper we set out to derive relations between discrete Painlevé equations which do not possess a free parameter. As explained in the introduction, the notion of absence of free parameter is a subtle one since we are referring to those parameters the values of which can be modified by autoBäcklund/Schlesinger transformations, to be distinguished from those that can be removed simply by the choice of the proper scaling. In the case of continuous Painlevé equations two parameterless ones exist, Painlevé I and the special case of Painlevé III where the terms cubic and inversely proportional in the dependent variable are absent.

Four basic zero-parameter discrete equations have been obtained using a coalescence degeneration procedure starting from equations related to the affine Weyl group $A_{1}^{(1)}+A_{1}^{(1)}$. However these four do not exhaust the list of all parameterless discrete Painlevé equations. Several more do exist, including three which can only be written in what we call a strongly asymmetric form. For all those equations we have presented the Miura transformations which allow one to transform one equation into another. Finally we have presented the continuous limits for the four basic parameterless equations showing that they are the discrete analogues of the parameterless continuous ones. The interesting result is that for two of these equations it is possible to find limits to both $\mathrm{P}_{\mathrm{I}}$ and the zero-parameter $\mathrm{P}_{\mathrm{III}}$.

One interesting feature of parameterless continuous Painlevé equations is that there exist Miura transformations which lead to equations where the second derivative does not enter linearly. Some of these equations belong to those classified by Cosgrove [16] but more complicated cases do also exist. Finding the discrete analogues of these equations and the corresponding Miura transformations could be the object of a future study. 


\section{References}

[1] E.L. Ince, Ordinary Differential Equations, Dover, London, (1956).

[2] C. Cosgrove, "Corrections and annotations to E.L. Ince, Ordinary differential equations, chapter 14, on the classification of Painlevé differential equations", unpublished, (1992).

[3] A. Ramani, B. Grammaticos, T. Tamizhmani and K.M. Tamizhmani, J. Phys. A 33 (2000) 579.

[4] Y. Ohyama, H. Kawamuko, H. Sakai and K. Okamoto, J. Math. Sci. Univ. Tokyo 13 (2006) 145.

[5] B Gambier, Acta Math. 33 (1909) 1.

[6] T. Tsuda, K. Okamoto and H. Sakai, Math. Ann. 331 (2005) 713.

[7] H. Sakai, Commun. Math. Phys. 220 (2001) 165.

[8] A. Ramani and B. Grammaticos, Physica A 228 (1996) 160.

[9] M. Jimbo and T. Miwa, Physica D 2 (1981) 407.

[10] B. Grammaticos, T. Tamizhmani, A. Ramani, A. S. Carstea and K.M.Tamizhmani, J. Phys. Soc. Japan 71 (2002) 443.

[11] M.D. Kruskal, K.M. Tamizhmani, B. Grammaticos and A. Ramani, Reg. Chao. Dyn. 5 (2000) 273.

[12] A. Fokas, B. Grammaticos and A. Ramani, J. of Math. Anal. and Appl. 180 (1993) 342.

[13] A. Ramani, B. Grammaticos and T. Tamizhmani, J. Phys. A 33 (2000) 3033.

[14] B. Grammaticos, A. Ramani, K.M. Tamizhmani, T. Tamizhmani and J. Satsuma, J. Math. Phys. 55 (2014) 053503.

[15] G.R.W. Quispel, J.A.G. Roberts and C.J. Thompson, Physica D 34 (1989) 183.

[16] C. Cosgrove and G. Scoufis, Stud. Appl. Math. 88 (1993) 25. 